\title{
Grey Literature Relating to Systematic Review
}

\begin{abstract}
Although the systematic review only demonstrated 27 papers, there is a wealth of conference abstracts. This indicates how important this topic is in being addressed. The advantages of reviewing the literature are that a much broader view is gained. However, the limitation is that it is only the published abstracts that are accessible. The abstracts themselves are also limited in the message that they convey.
\end{abstract}

